\title{
26476 - HEMODYNAMIC CHANGES DURING OFF-PUMP CORONARY ARTERY BYPASS GRAFTING
}

\section{Ashraf Fayad FCARCSI FACC, Homer Yang, University Of Ottawa, Ottawa, ONTARIO, Canada}

Introduction:

Significant hemodynamic changes often occur during surgical displacement of the heart in off-pump coronary artery bypass grafting (OPCAB) (1). These changes involve mainly arterial and venous pressures, intraventricular volumes, cardiac out put $(\mathrm{CO})$ and mixed venous oxygen saturation $(\mathrm{SvO} 2 \%)$. The purpose of this study is to document the hemodynamic changes during the periods when the heart is tilted in patients undergoing $\mathrm{OPCAB}$ and examine their post-operative outcomes.

Methods:

This is an observational study in which, 34 consecutive patients were consented after obtaining the local ethical approval. Patients were excluded if the decision was made intraoperatively to go on cardiopulmonary bypass $(\mathrm{CPB})$. All patients received the standard anesthetic monitoring, arterial line and Swan-Ganz CCOmbo V catheters connected to a vigilance monitor. Surgical and anesthetic techniques were as per institutional standard. The use of intraoperative inotropes, vassopressors or vasodilatation was measured against patient's blood pressure. Preoperative patients demography and comorbid diseases were documented. The intraoperative hemodynamic variables were collected before and during heart positioning for anastomosis of the grafts. Postoperative outcome were retrospectively reviewed in each patient. Hemodynamic variables in this study included heart rate, Systemic and pulmonary pressure, right ventricular end diastolic volume (RVEDV), cardiac output (CO) and mixed venous saturation were documented every five minutes during anastomosis of the grafts. Post-operative outcomes were identified as length of ventilation, length of stay, post-op arrhythmias, myocardial ischemia (MI), renal impairment and mortality.

Results:

Eighteen patients (53\%) experienced intraoperative hemodynamic instability during the initial heart positing or testing and the surgical decision was made to go on CPB. The remaining 16 patients ( 9 males, 7 females; mean age, $68.9 \pm 8.7$ years) completed the study with no perioperative mortality. Number of grafts ranged between $2-4$ ( $63 \%$ had 3 grafts), involved the right coronary artery (RCA), circumflex (CX) and the left ascending artery (LAD) with average occlusion time of $17.0 \pm 7.5,22.5 \pm 5.9$ and $16.5 \pm 4.7$ respectively. There was a reduction in the RVEDV, $\mathrm{CO}$ and mixed venous oxygen saturation during heart positioning in all grafts. However, positioning for the circumflex artery showed the greatest reduction in the previous parameters. Postoperative complications were high $(87.5 \%)$ and significant in patients who had $\mathrm{cx}$ artery grafting. Discussion:

Hemodynamic instability occurs mainly during $\mathrm{cx}$ artery grafting with significant postoperative complications. This study confirms the previous findings regarding the significant hemodynamic changes that occur during the lateral wall vessel grafting (2-3). Selection criteria for OPCAB may need to be revisited if the cx artery is involved. 